Classification

Physics Abstracts

$42.60 \mathrm{~K}-46.30 \mathrm{M}-61.80 \mathrm{~B}$

\title{
Déformation à l'échelle cristallographique d'alliages à base de nickel mono- et polycristallins par choc laser en mode confiné
}

\author{
P. Forget $\left(^{*}\right)$ et $M$. Jeandin \\ Ecole Nationale Superieure des Mines de Paris, Centre des Matériaux P.M. Fourt, B.P. 87, 91003 \\ Evry Cedex, France
}

(Reçu le 17 février 1995, révisé le 7 avril 1995, accepté le 17 mai 1995)

\begin{abstract}
Résumé. - Le traitement de "grenaillage photonique" par choc laser vise à écrouir un matériau et à y introduire des contraintes résiduelles de compression en vue d'améliorer ses propriétés mécaniques. Dans le cas de superalliages aéronautiques à base de nickel, on attend une augmentation de la tenue en fatigue. On traite ici de la déformation induite par le choc grâce à une étude microstructurale fondée sur l'observation des microreliefs de surface dans la zone traitée. Ceux-ci sont interprétés comme le résultat de l'évolution cristallographique du matériau soumis au choc. Les phénomènes de glissement, sont, en particulier, précisément identifiés. Plus généralement, les hétérogénéités de surface dans les différentes parties de l'impact sont expliquées à la lumière de résultats sur la propagation des ondes de détente de surface et sur la création de contraintes résiduelles. Deux superalliages à base de nickel, l'AM1 monocristallin et l'Inconel 718 polycristallin, ont été étudiés.
\end{abstract}

\begin{abstract}
The so-called "laser peening" treatment based on laser shock processing has been developed to improve mechanical properties due to work-hardening and formation of residual stresses. When applied to aeronautical Ni-based superalloys, an increase of fatigue resistance is expected. This article deals with deformation resulting from laser shock. The study is centered on the observation of superficial micro-roughness in the impacted zone. Micro-roughness is discussed as resulting from crystallographic changes due to shock process. Various slip phenomena were in particular determined. More generally, superficial heterogeneities in the different parts of the shocked area are discussed in the light of results on release wave propagation and formation of residual stresses. Two Ni-based superalloys, i.e. AM1 single crystal and polycrystalline Inconel 718 , were used.
\end{abstract}

\section{Introduction}

Si le principe du traitement par choc laser pour le grenaillage dit "photonique" de pièces remonte au début des années quatre-vingt [?], son application aux superalliages de nickel aéronautiques n'est que récente $[2,3]$. Il s'agit principalement d'augmenter la résistance à la

$\left(^{*}\right)$ Adresse actuelle: Centre d'Etudes Nucléaires de Saclay, 91191 Gif-sur-Yvette Cedex, France 
Tableau I. - Composition chimique des alliages, \% pds.

[Chemical composition of the alloys, Wt.\%.]

\begin{tabular}{cccccccccccc}
\hline Alliage & $\mathrm{Co}$ & $\mathrm{Cr}$ & $\mathrm{Mo}$ & $\mathrm{Fe}$ & $\mathrm{W}$ & $\mathrm{Ti}$ & $\mathrm{Ta}$ & $\mathrm{Nb}$ & $\mathrm{C}$ & $\mathrm{B}$ & $\mathrm{N}$ \\
\hline \multirow{2}{*}{ AM1 } & 6.5 & 75 & 2.0 & & 5.5 & 1.2 & 8.0 & & & & Bal. \\
INCONEL 718 & 0.5 & 18.0 & 2.9 & 18.1 & & 0.9 & & 5.2 & 0.030 & 0.003 & Bal. \\
\hline
\end{tabular}

fatigue oligocyclique de composants tels que les disques (alvéoles) et les aubes (pieds) de turbine grâce à l'écrouissage du matériau en profondeur et à l'introduction de contraintes résiduelles superficielles de compression.

Au delà de l'étude des paramètres d'irradiation laser et de la caractérisation mécanique des matériaux traités, la compréhension du procédé passe par une phase de modélisation des contraintes résiduelles et par l'étude de la déformation induite par le choc. C'est de cette dernière que cet article traite en particulier. Certains aspects y sont cependant discutés à la lumière de résultats de la modélisation préalablement mentionnée $[4,5]$ qui constituait un autre volet de l'étude générale dont est issu ce travail.

La déformation par choc laser appliqué aux superalliages n'a été jusqu'ici l'objet que d'études plutôt consacrées aux mécanismes observables à l'échelle de la microscopie à transmission $[2,3,6,7]$ ou à l'échelle de l'impact laser [3]. Cette étude s'attache aux phénomènes microscopiques intéressant la cristallographie. Elle repose sur l'observation, principalement en microscopie optique, de microreliefs de surface (préalablement polie "miroir") formés au cours du choc puis sur leur interprétation en tant que résultat de différents phénomènes de glissement. Deux types de superalliages à base de nickel ont été choisis. L'un, l'AM1, était monocristallin solidifié suivant différentes orientations. Les différents directions de glissement s'y trouvent en quelque sorte "discrétisées". De plus, la structure dendritique de cet alliage introduit un terme d'hétérogénéité dont il était bon d'étudier l'influence. L'autre alliage a été pris polycristallin à grains suffisamment gros pour mettre en évidence des structures de déformation : il s'agissait de l'Inconel 718, pour lequel les études de déformation les plus récentes se situaient dans le domaine du forgeage [8] et non dans celui des traitements de surfaces.

\section{Matériaux et méthodes}

2.1. Matériaux. - Les matériaux étudiés étaient des superalliages de fonderie à base de nickel : l'alliage AM1 monocristallin et l'Inconel 718 (NC19FeNb selon la norme AFNOR) dont les compositions sont dans le tableau I. Ils sont constitués tous deux d'une matrice austénitique $\gamma$ de structure cubique à faces centrées. Dans le cas de l'AM1, la matrice est durcie par précipitation d'une phase quasi cohérente et ordonnée $\gamma^{\prime} \mathrm{Ni}_{3}(\mathrm{Al}, \mathrm{Ti})$ de structure cubique simple $\mathrm{L1}_{2}$. Le durcissement de l'Inconel 718 résulte de la précipitation de $\gamma^{\prime}\left(\mathrm{L1}_{2}\right)$ et $\gamma^{\prime \prime}\left(\mathrm{DO}_{22}\right)$ lors de son élaboration par coulée puis forgeage et des traitements thermiques mentionnés dans le tableau II.

Les échantillons d'AM1 ont été extraits de barreaux de diamètre $\oslash 10$ ou $\oslash 13 \mathrm{~mm}$ élaborés par la méthode de Bridgman selon différentes directions de solidification correspondant à l'axe du barreau : [001], [111] et [110]. 
Tableau II. - Traitements thermqques des alliages.

[Heat treatments applied to the alloys.]

\begin{tabular}{|c|c|c|}
\hline Trattement & $\mathrm{AMI}$ & Inconel 718 \\
\hline $\begin{array}{l}\text { Remise en solution } \\
\left(\gamma^{\prime} \text { et } \gamma^{\prime \prime}\right)\end{array}$ & $\begin{array}{c}1.3(1) 1^{\circ} \mathrm{C}-3 \mathrm{~h} \\
\text { trempe air (t.a.) }\end{array}$ & $955^{\circ} \mathrm{C}-1 \mathrm{~h} / \mathrm{tad}$ \\
\hline $\begin{array}{l}\text { Précipitation } \\
\text { des } \gamma^{\prime} \text { et } \gamma^{\prime \prime}\end{array}$ & $\begin{aligned} & 11000^{\circ} \mathrm{C}-5 \mathrm{~h} / \mathrm{t} . \mathrm{a} \\
+\quad & 8700^{\circ} \mathrm{C}-16 \mathrm{~h}\end{aligned}$ & $\begin{aligned} & 720^{\circ} \mathrm{C}-8 \mathrm{~h} \text { (refiord. à } \\
\leq & 50^{\circ} \mathrm{C} / \mathrm{h} \text { jusquà } 620^{\circ} \mathrm{C} \text { ) } \\
+ & 620^{\circ} \mathrm{C}-8 \mathrm{~h} / \mathrm{ta} .\end{aligned}$ \\
\hline
\end{tabular}

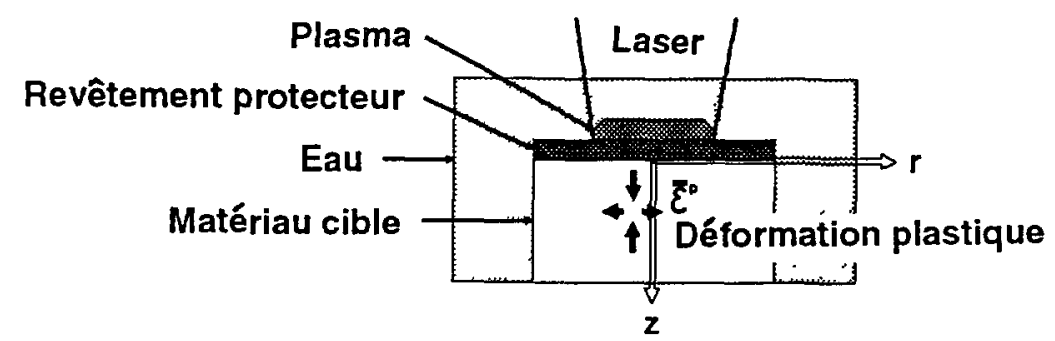

Fig. 1. - Principe du choc laser, coordonnées cylindriques.

[Laser shock principle (cylindrical coordinates).]

2.2. CHOC LASER. - Le choc laser consiste à irradier un matériau pendant un temps très court afin d'obtenir une forte densité de puissance lumineuse sur la surface "impactée". Une fine couche de matière est vaporisée et forme un plasma de pression très élevée $[1,2]$. Cette pression se transmet au matériau sous la forme d'une onde de choc qui se propage en profondeur ; elle est maintenue jusqu'à la détente du plasma. En recouvrant le matériau d'une couche transparente comme de l'eau, cas de cette étude, on limite la détente du plasma alors confiné. La pression appliquée par le plasma et son temps de maintien s'en trouvent augmentés, d'un facteur de 1 à 10 pour la pression et d'environ 3 pour le temps de maintien.

On a utilisé :

- le laser multifaisceau du Laboratoire d'Utilisation des Lasers Intenses ("L.U.L.I.") de l'Ecole Polytechnique/Palaiseau qui permet de délivrer des impacts de durée $\tau_{\mathrm{e}} \approx 30 \mathrm{~ns}$ pour une énergie maximale par impact de $150 \mathrm{~J}, \tau_{\mathrm{e}} \approx 3$ ns pour une énergie maximale par impact de $100 \mathrm{~J}, \tau_{\mathrm{e}} \approx 600 \mathrm{pns}$, en mode bloqué, pour une énergie maximale de $80 \mathrm{~J}$ par impact ;

- le laser du Laboratoire d'Application des Lasers de Puissance ("L.A.L.P.") de l'unité mixte C.N.R.S.-E.T.C.A./Arcueil délivrant des impacts de durée $\tau_{\mathrm{e}} \approx 20$ à $30 \mathrm{~ns}$ sur deux faisceaux pour une énergie maximale de $2 \times 80 \mathrm{~J}$.

Au cours de cette étude, les temps d'impulsion ont été choisis dans la gamme 17-40 ns pour des impacts de diamètre $8 \mathrm{~mm}$ obtenus en mode confiné. Un revêtement absorbant en ruban adhésif noir recouvrait préalablement les surfaces à traiter pour les protéger de la fusion, des contraintes de traction associées et générer le plasma (Fig. 1). 
2.3. OBSERVATION DES SURFACES TRAITÉES. - Les micro-reliefs de surface ont été étudiés par microscopie optique. Cette dernière a été préférée à la profilométrie classique peu appropriée au repérage et la détection de reliefs peu marqués, nombreux et présents sur une large zone correspondant à la taille de l'impact.

On a fait appel à une technique de microscopie optique utilisant une binoculaire "WILD MAKROSKOP M420" selon une procédure d'observation développée à l'Ecole des Mines de Paris par l'un des auteurs (P. Forget). Celle-ci repose sur la discrimination des rayons réfléchis par les reliefs de l'échantillon. Cette discrimination résulte de la sélection des rayons appartenant à un cône lumineux dit "cône utile" qui parviennent à traverser toute la colonne optique jusqu'à l'oculaire, le reste étant absorbé par les parois de la colonne. Pour les plus forts grandissements, les observations ont été effectuées sur échantillons inclinés pour accroître le phénomène de discrimination ; l'augmentation de l'angle d'inclinaison et du grandissement crée une illusion d'optique se traduisant par l'impression inversée du relief. Ce phénomène ne fausse en rien les interprétations et s'explique aisément par des considérations optiques qui ne seront pas développées ici.

Cette technique s'est révélée aussi supérieure à la microscopie optique confocale qui a été tentée mais sans grand succès aussi bien avec un équipement à disque tournant qu'avec un autre à balayage laser. Les dénivelés à détecter étaient en effet à pentes trop faibles (moins de $\left.1^{\circ}\right)$.

\section{Déformation microscopique des alliages AM1 et Inconel 718}

3.1. DÉformation du monocristal AM1. - Parmi les orientations du monocristal étudiées, l'orientation [001], normale à la surface irradiée de l'échantillon, présente l'avantage de respecter au maximum la symétrie du matériau lors du choc. On fait en effet coïncider la direction de solidification, axe des dendrites primaires, avec la direction de propagation de l'onde de choc.

La première constatation est l'effet de poinçonnement dû au choc sur le matériau qui d'ailleurs se produit quelqu'en soit la nature. On a constaté aussi (Fig. 2) l'effet de la structure de solidification sur la déformation plastique par choc. Les dendrites, plus dures que l'espace interdendritique, ont été moins déformées par l'onde de choc. Est ainsi clairement mise en lumière cette évidence : la réponse du matériau à la sollicitation est fortement liée à ses caractéristiques mécaniques, en particulier à sa limite d'élasticité.

L'apparition de "rayures" après le choc atteste aussi cette conclusion. Sur l'échantillon initialement poli "miroir", des rayures couvrent toute la surface impactée, à l'exception des sousintensités consécutives aux hétérogénéités de l'éclairement de la zone irradiée dues en particulier à la diffusion locale du faisceau par des particules de poussière, et ne s'étendent pas en dehors de l'impact (Fig. 2). Etant situées en contrebas par rapport à la surface moyenne de l'échantillon, elles ne peuvent avoir été créées ni avant le choc, ni après. Elles résultent donc du choc lui-même. Bien que le polissage ait éliminé la trace de toutes les rayures antérieures au choc, celles-ci avaient néanmoins affecté le matériau en profondeur, de manière invisible, par une augmentation de l'écrouissage. La modification associée du critère de plasticité se traduit au cours du choc par une différence du niveau de déformation plastique entre l'emplacement de la rayure et les alentours. On notera d'une part que ces rayures sont en creux, d'autre part qu'elles sont quasiment invisibles par microscopie optique classique. Seule une méthode d'observation du type de celles employées dans cette étude (cf. paragraphe 2.3.) a permis de les révéler.

A plus fort grandissement, on observe que la décoration de la structure dendritique par le choc se traduit par l'apparition, dans l'espace interdendritique, de petits carrés de 20 à $80 \mu \mathrm{m}$ 


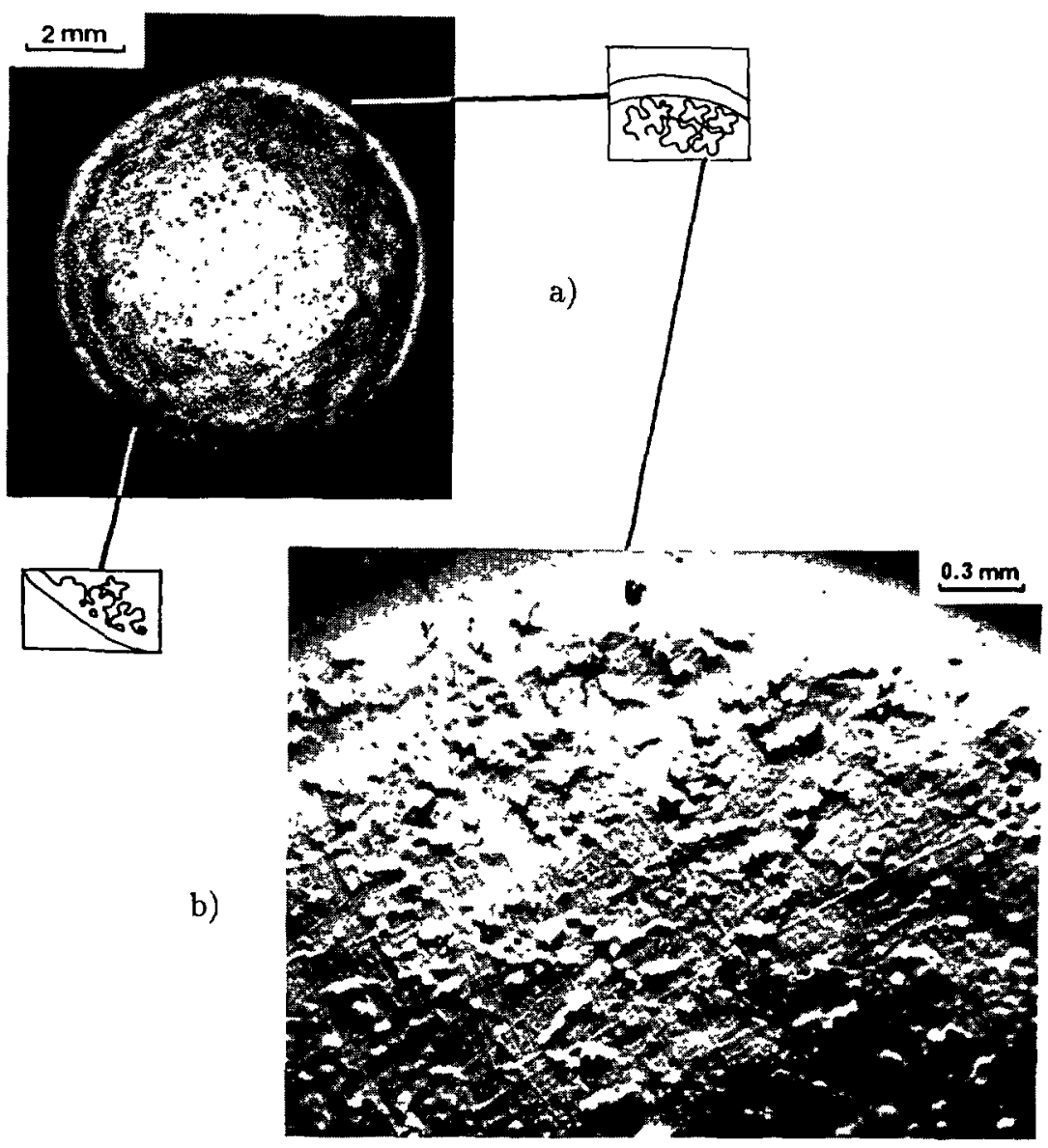

Fig. 2. - Surface d'un échantillon d'AM1 d'orientation [001] après un choc laser confiné, $\tau_{e}$ (temps d'impulsion) $=40 \mathrm{~ns}, d_{\mathrm{p}}$ (densité de puissance) $=5,4 \mathrm{GW} \cdot \mathrm{cm}^{-2}$, Laser L.U.L.I.. Micrographie optique. Révélation de la structure dendritique (vue générale a) et des systèmes de glissement (détail b), avec inversion de relief par illusion d'optique).

[[001] AM1 sample surface after a confined laser shock (pulse length: 40 ns, power density $=5.4$ GW.cm ${ }^{-2}$, "L.U.L.I." laser). Optical micrograph showing a dendritic structure, a) general view b) slip systems (inverted relief due to an optical illusion).]

de côté (Fig. 2). Les mêmes figures ont été observées sur des échantillons monocristallins d'Astroloy ou de superalliage à base de nickel Waspaloy [6] et des figures similaires (étoiles à 6 branches) ont été mises en évidence en bordure d'impacts laser ponctuels sur des échantillons de cuivre [9].

Les observations sont interprétées en fonction de l'activation possible des systèmes de glissement par l'onde plane pour les 3 orientations du monocristal choisies (Tab. III).

Les côtés des carrés correspondent à des directions cristallographiques [110] et [110], c'est à dire aux traces des plans denses $(111),(\overline{1} \overline{1} 1),(1 \overline{1} \overline{1})$ et $(\overline{1} 1 \overline{1})$ sur la surface (001) (Fig. 3a). Dans le cas d'échantillons d'orientation [111], apparaissent non pas des carrés mais, en moins grande

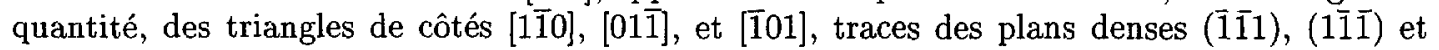


Tableau III. - Actrvation des systèmes de glissements par l'onde plane pour les 3 orientatrons monocristallines [001], [110] et [111].

[Activation of the slip systems due to the plane wave for the 3 orientations of the single crystal, i.e. [001], [110] and [111].]

\begin{tabular}{|c|c|c|c|c|}
\hline Echantillon & Trace & Plan de glissement & Angle de la surface & Actuvatior \\
\hline \multirow{4}{*}[001]{} & \multirow[b]{2}{*}[\begin{array}{lll}{1}&{1}&{0}\end{array}]{} & $\left(\begin{array}{lll}1 & 1 & 1\end{array}\right)$ & $54^{\circ}$ & oui \\
\hline & & $\left(1 \frac{1}{1}\right)$ & $54^{\circ}$ & our \\
\hline & \multirow{2}{*}[1\overline{1}0]{} & $(\overline{1} \overline{1})$ & $54^{\circ}$ & ouj \\
\hline & & $(\overline{1} 1 \overline{1})$ & $54^{\circ}$ & ous \\
\hline \multirow{4}{*}[\begin{array}{lll}{1}&{1}&{1}\end{array}]{} & --- & $\left(\begin{array}{lll}1 & 1 & 1\end{array}\right)$ & $0^{\circ}$ & non \\
\hline & {$[1 \overline{1} 0]$} & $(\overline{1} \overline{1} 1)$ & $70^{\circ}$ & ou1 \\
\hline & {$[01 \overline{1}]$} & $(1 \overline{1} \overline{1})$ & $70^{\circ}$ & oul \\
\hline & {$[\overline{1} 01]$} & $(\overline{1} 1 \overline{1})$ & $70^{\circ}$ & oui \\
\hline \multirow{4}{*}[\begin{array}{lll}{1}&{1}&{0}\end{array}]{} & \multirow{2}{*}{$\begin{array}{l}1 \overline{1} 0] \\
-\end{array}$} & $\left(\begin{array}{lll}1 & 1 & 1\end{array}\right)$ & $35^{\circ}$ & ous \\
\hline & & $(\overline{1} \overline{1} 1)$ & $35^{\circ}$ & our \\
\hline & {$[1 \overline{1} 2]$} & $(1 \overline{1} \overline{1})$ & $90^{\circ}$ & non \\
\hline & {$\left[\begin{array}{l}\overline{1} \\
12\end{array}\right]$} & $(\overline{1} 1 \overline{1})$ & $90^{\circ}$ & non \\
\hline
\end{tabular}

(11T) sur la surface (111) (Fig. 3b). En revanche, dans le cas d'échantillons d'orientation [110],

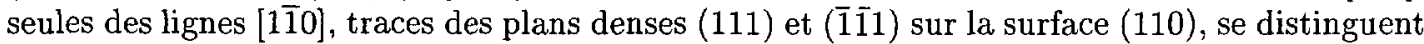
nettement (Fig. 3c). Elles sont éventuellement bordées de chaque côté de petits segments [112] et [112], traces des plans denses $(1 \overline{1} \overline{1})$ et $(\overline{1} 1 \overline{1})$ sur la surface (110). Très exceptionnellement, des demi-losanges ( 2 côtés et la petite diagonale) ont été créés en frontière de l'impact (Fig. 3d).

Cela n'est qu'une manifestation du caractère uniaxial de la déformation introduite par l'onde de choc. Pour un chargement en déformation uniaxiale correspondant à un tenseur de contraintes :

$$
\overline{\bar{\sigma}}=\left[\begin{array}{ccc}
\sigma_{r r} & 0 & 0 \\
0 & \sigma_{r \tau} & 0 \\
0 & 0 & \sigma_{z z}
\end{array}\right]
$$

le cisaillement maximal est exercé dans les plans à $45^{\circ}$ de la surface (Fig. 4). Ainsi, dans le cas des échantillons [001], les 4 plans de glissement, à $54^{\circ}$, ont été fortement activés. Pour les échantillons [111], 3 plans de glissement, à $70^{\circ}$, ne le furent que moyennement . Pour les échantillons [110], les 2 plans de glissement à $35^{\circ}$ ont été activés, tandis que les 2 plans à $90^{\circ}$ ne le furent pas. Les quelques demi-losanges complets dénotent un faible écart au chargement uniaxial à la périphérie de l'impact. On pourrait vérifier que les phénomènes observés obéissent à la loi de Schmid qui définit l'ensemble des systèmes de glissement facile.

Les mesures par microscopie optique, selon la procédure mise au point dans le cadre de cette étude (cf. paragraphe 2.3.), ont montré que les frontières des carrés, pour les échantillons [001] constituaient des surfaces de quelques micromètres de largeur inclinées à $20 \times 10^{-3} \mathrm{rd}$, soit $1^{\circ}$, par rapport à l'horizontale (l'erreur est estimée à un facteur 2). Les carrés sont donc des creux de $50 \mathrm{~nm}$ de profondeur seulement, ce qui laisse penser que les rayures, au contraste plus faible, sont encore moins profondes. Toutefois, s'il a été possible de déterminer le dénivelé 


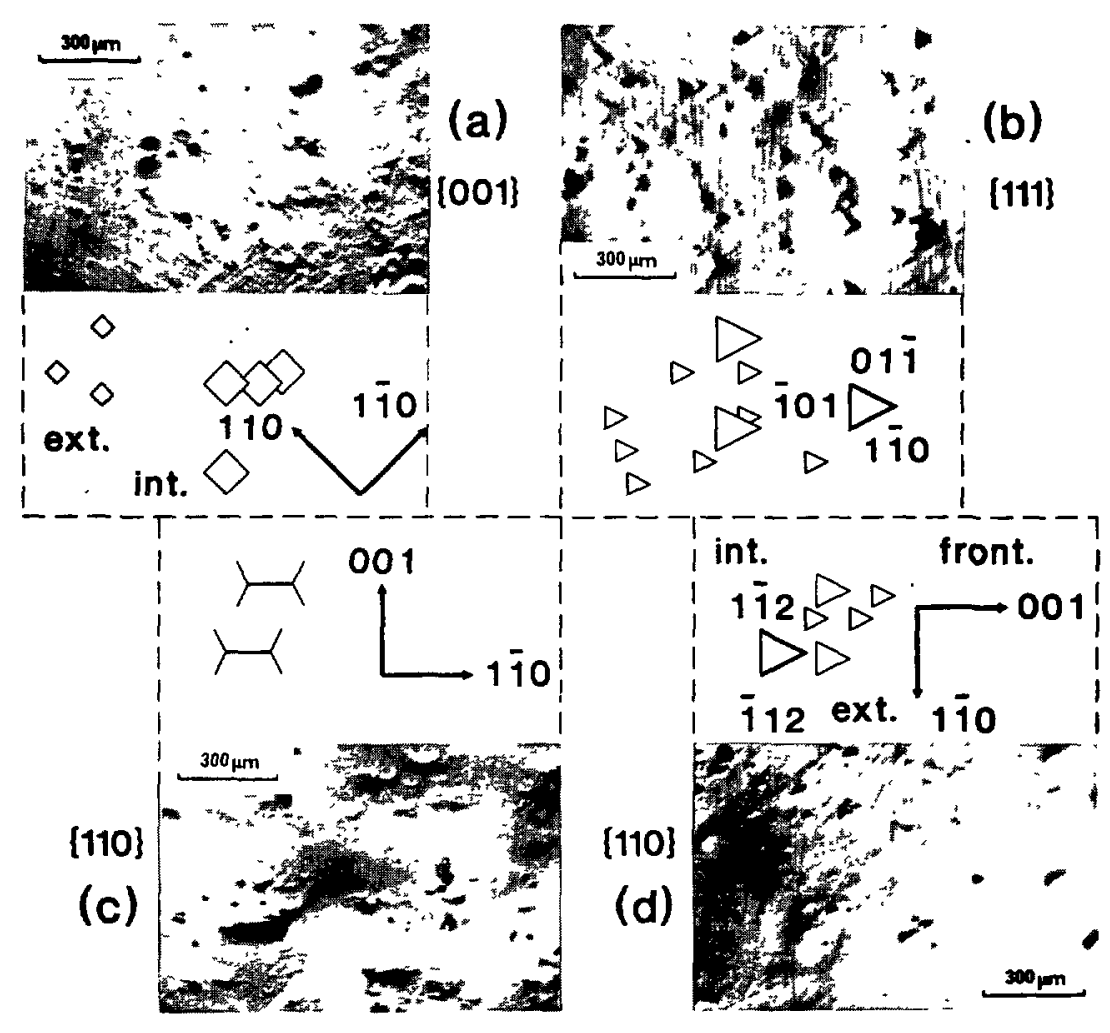

Fig. 3. - Surfaces d'échantillons d'AM1 de différentes orientations après chocs laser confinés (laser L.A.L.P.) : révélation des systèmes de glissement. Micrographies optiques avec inversion de relief par illusion d'optique. a) Echantillon d'orientation [001], $\tau_{\mathrm{e}}=22 \mathrm{~ns}, d_{\mathrm{p}}=8,2 \mathrm{GW} \cdot \mathrm{cm}^{-2}$, b) Echantillon d'orientation [111], $\tau_{e}=17 \mathrm{~ns}, d_{\mathrm{p}}=10,7 \mathrm{GW} . \mathrm{cm}^{-2}$, c) Echantillon d'orientation [001], $\tau_{\mathrm{e}}=21 \mathrm{~ns}$, $d_{\mathrm{p}}=16,9 \mathrm{GW} \cdot \mathrm{cm}^{-2}$, d) Bordure d'échantillon d'orientation [110], $\tau_{\mathrm{e}}=22 \mathrm{~ns}, d_{\mathrm{p}}=8,4 \mathrm{GW} . \mathrm{cm}^{-2}$.

[AM1 superficial aspect for various orientations after confined laser shocks (using the "L.A.L.P." laser) exhibing slip systems. Optical micrographs with relief inversion due to an optical illusion. a) [001] sample, pulse length $=22 \mathrm{~ns}$, power density $\left.=8.2 \mathrm{GW} \cdot \mathrm{cm}^{-2}, \mathrm{~b}\right)$ [111] sample, pulse length $=$ $17 \mathrm{~ns}$, power density $=10.7 \mathrm{GW} \cdot \mathrm{cm}^{-2}$, c) [001], sample, pulse length $=21 \mathrm{~ns}$, power density $=169$ GW.cm ${ }^{-2}$, d) Rim of $[110]$ sample, pulse length $=22 \mathrm{~ns}$, power density $\left.=8.4 \mathrm{GW} \cdot \mathrm{cm}^{-2}\right]$
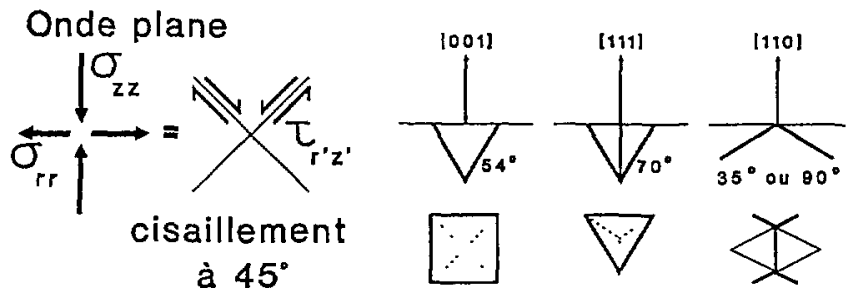

Fig. 4. - Activation des systèmes de glissements par l'onde plane pour les 3 orientations monocritallines [001], [110] et [111].

[Slip system activation due to the plane wave for [001], [110] and [111] single crystal orientatıons.] 


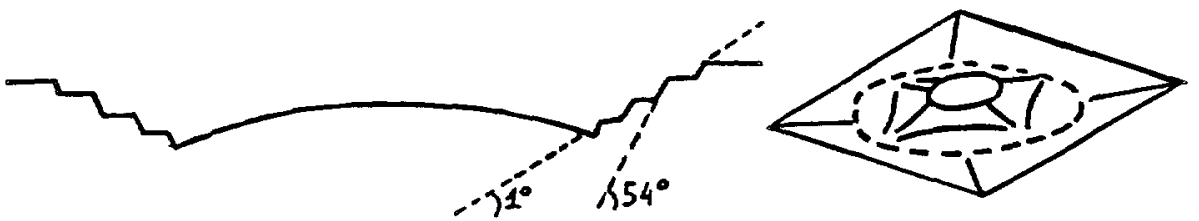

Fig. 5. - Estimation des reliefs des carrés activés par l'onde plane sur échantillons monocristallins d'orientation [001].

[Estimation of the dimension of squares activated by the plane wave for [001] single crystal].

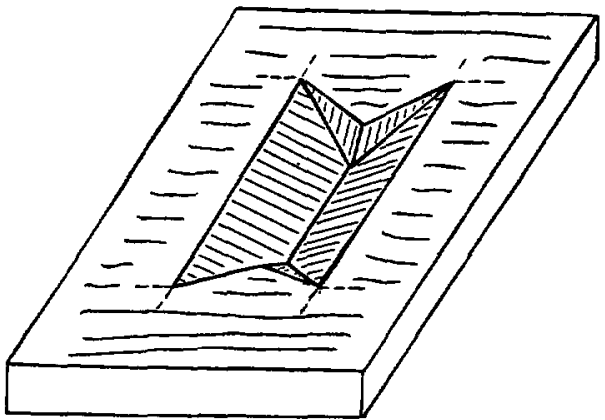

Fig. 6. - Aspect des vallées activées par l'onde plane sur échantillons monocristallins d'orientation [110].

[Aspects of the valleys activated by the plane wave for [110] single crystal.]

à la frontière des carrés, il aurait été hasardeux de tenter de mesurer le profil en leur centre. Il semble néanmoins, d'après les contrastes les mieux définis, que la surface soit bombée au centre, ce qui irait dans le sens de la conservation de volume lors de la déformation plastique (Fig. 5).

Pour les échantillons [111], les reliefs des triangles obéissent aux mêmes tendances ; le relief à l'intérieur des triangles était cependant rarement discernable.

Il en est tout autrement dans le cas des échantillons [110] en dehors des demi-losanges, puisque les traces des plans de glissement ne définissaient pas des figures formées. Elles constituaient alors des vallées parallèles se raccordant au "plateau" correspondant au niveau moyen de la surface de l'impact, par l'intermédiaire des "failles" constituées par les segments [1̄̄2] et $[\overline{1} 12]$, traces des plans verticaux $(1 \overline{1} \overline{1})$ et $(\overline{1} 1 \overline{1})$. Ces plans de glissement n'ont donc pas été activés par l'onde plane elle-même mais par l'incompatibilité engendrée par les plans (111) et (1111) (Fig. 6).

3.2. DÉformation DU POLYCRISTAL INCONEL 718. - Le choc sur monocristal permet de visualiser la déformation plastique (et non ses seuls effets sur le relief) mais il comporte l'inconvénient de la forte dépendance de celle-ci vis-à-vis de l'hétérogénéité inhérente au matériau. Pour obtenir une visualisation semi-quantitative de la déformation plastique, il faut utiliser un matériau homogène à l'échelle des hétérogénéités de chargement et isotrope, donc un matériau polycristallin mais dont les grains soient suffisamment gros pour pouvoir mettre en évidence des structures de déformation. 
Le traitement d'échantillons d'Inconel 718 polycristallin à gros grain répondait à ces conditions. La déformation plastique s'y est manifestée de deux manières : par décoration des joints de grains lorsque ceux-ci glissaient les uns par rapport aux autres (du fait des marches entre grains) et par l'apparition de lignes de glissement plus ou moins fortement marquées à l'intérieur des grains.

Après choc confiné, on peut distinguer 5 domaines en surface sur l'Tnconel 718 (Fig. 7) :

- Sur la majeure partie de l'impact, à l'intérieur d'une couronne située à une certaine distance de la frontière et excluant le centre, la déformation est à peu près homogène et modérée. Elle se traduit par la décoration des grains avec une activation faible d'un, voire deux systèmes de glissement, à l'intérieur des grains.

- Au centre de l'échantillon sur une surface inférieure à $1 \mathrm{~mm}^{2}$, un seul système de glissement a été très nettement activé ou chaque grain. Cette augmentation de la déformation plastique est suffisamment nette pour être visible en microscopie optique classique ; elle correspond à une hétérogénéité du niveau de contrainte mise en évidence par diffraction $X$ dans un autre volet de cette étude [4]. Cette hétérogénéité se traduit par un creux dans le profil de contrainte de compression à peu près uniforme dans la zone impactée. Ce creux se produit quel que soit le laser et quel qu'en soit le réglage. Il n'est donc pas attribuable à une sous-intensité du faisceau laser, qui ne présente pas de point froid en son centre. Cela est corroboré par le fait que les mesures de largeur de pics de diffraction, uniformes autour de $r=0$, ne correspondent pas à un affaiblissement de déformation plastique. Ainsi, la contrainte d'activation d'un système de glissement par grain au centre traduit un surcroît de déformation plastique cumulé. Par rapport au reste de l'impact, cette déformation plastique supplémentaire est, d'un point de vue tensoriel, d'un signe opposé à celle créée par l'onde plane, aboutissant à un déficit de contrainte de compression. Dans le cas de l'Astroloy (NK17 CDAT), superalliage de la famille de l'Inconel 718, le creux de contraintes disparaît très vite en profondeur. La contrainte $\sigma_{r r}$ rejoint quasiment la valeur moyenne à distance du centre dès une profondeur de l'ordre de $150 \mu \mathrm{m}$ [3]. La formation de ce creux de contraintes est donc un phénomène de nature essentiellement surfacique.

- Entre la couronne (1er domaine) et la frontière de l'impact, c'est-à-dire dans un domaine caractérisé par un fort poinçonnement du matériau (paragraphe 3.1.), les joints de grains sont plus fortement révélés qu'ailleurs. En effet, en bord d'impact, ce poinçonnement est venu s'ajouter au chargement d'onde plane, augmentant de ce fait l'amplitude de l'invariant de Von Misès $J_{2}^{\prime}$. A la déformation plastique du domaine précédent, créée par l'onde plane, du type $\varepsilon_{z z}^{p}=-2 \varepsilon_{r r}^{P}=-2 \varepsilon_{\theta \theta}^{P}$, est venue ici s'ajouter une déformation plastique de cisaillement, du type $\varepsilon_{r z}^{p}$. Ce cisaillement peut être évalué simplement par la mesure du dénivelé en frontière d'impact. Pour un enfoncement $\Delta u_{z} \approx 2,5 \mu \mathrm{m}$ sur une largeur de la paroi $\Delta r \approx 0,5 \mathrm{~mm}$ (valeurs caractéristiques pour un choc de $33 \mathrm{~ns}$ à 8,2 GW. $\mathrm{cm}^{-2}$, déterminées par rugosimétrie), le cisaillement plastique est :

$$
\varepsilon_{r z}^{p} \approx \frac{1}{2} \frac{\Delta u_{z}}{\Delta r} \approx-0,25 \%
$$

compatible avec les valeurs trouvées dans la modélisation [5].

- Sur une bande d'environ $1 \mathrm{~mm}$ de large à l'extérieur de l'impact, les joints de grains ont été légèrement révélés, de plus en plus faiblement à mesure que l'on s'éloigne de la frontière de l'impact. Or au cours du choc apparaît une discontinuité de chargement 


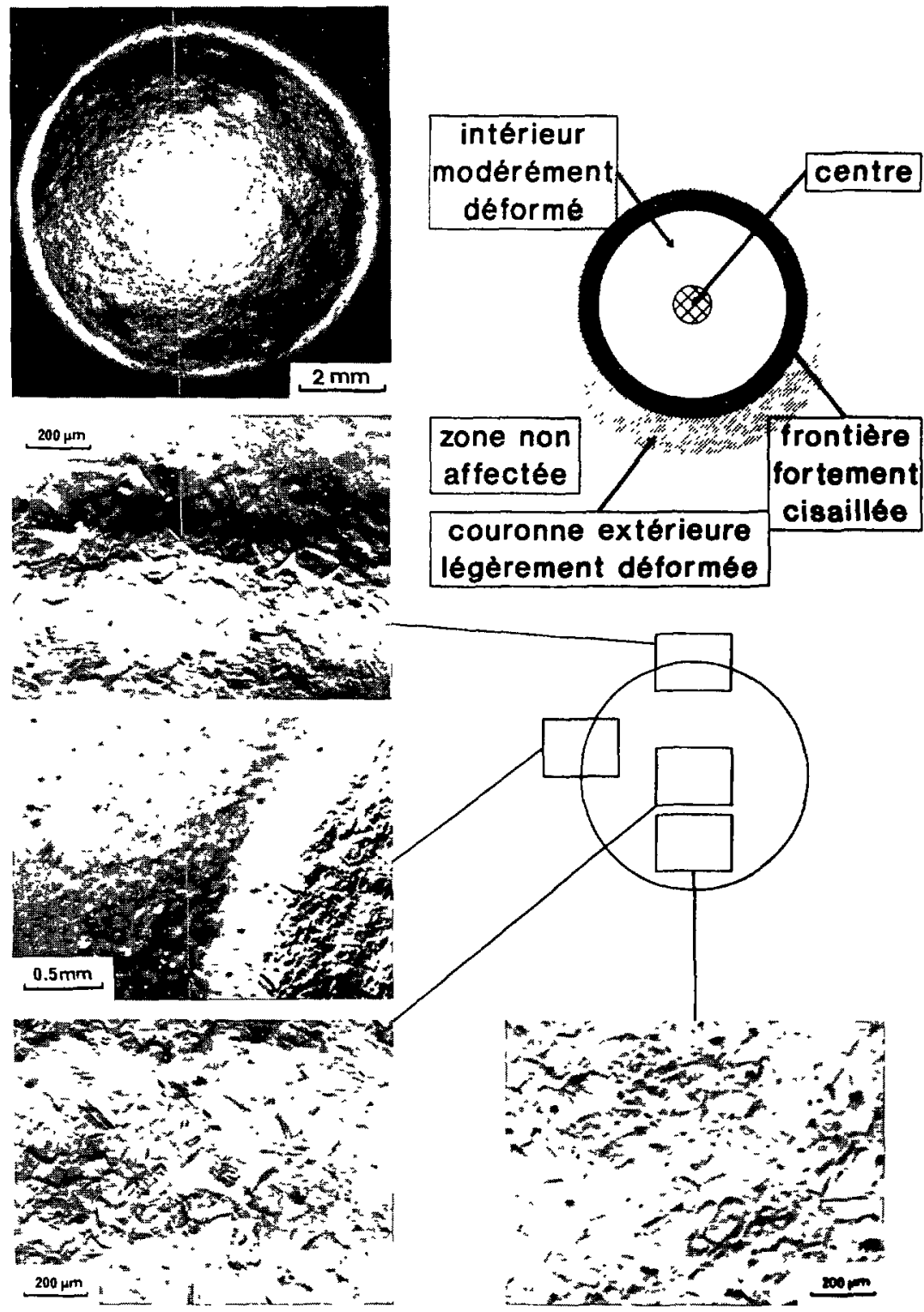

Fig. 7. - Surface d'échantillon d'Inconel 718 après un choc laser confiné $\left(\tau_{\mathrm{e}}=33 \mathrm{~ns}, d_{\mathrm{p}}=7,6\right.$ GW. $\mathrm{cm}^{-2}$, Laser L.U.L.I.) : localisation des 5 domaines de déformation.

[Inconel 718 sample surface after a confined laser shock (pulse length $=33 \mathrm{~ns}$, power density $=7.6$ GW.cm ${ }^{-2}$, "L.U.L.I." laser) showing the 5 typical deformation areas.]

entre l'intérieur de l'impact et l'extérieur. Cette discontinuité, qui n'est pas statiquement admissible, ne peut s'éliminer au cours du temps que par un effet de bord jouant sur la propagation d'onde. L'invariant $J_{2}^{\prime}$, initialement discontinu à la frontière de l'impact, devient lui aussi continu au cours du choc. On constate ici que cet effet de bord est suffisamment grand pour plastifier le matériau à l'extérieur de l'impact (Fig. 8). 


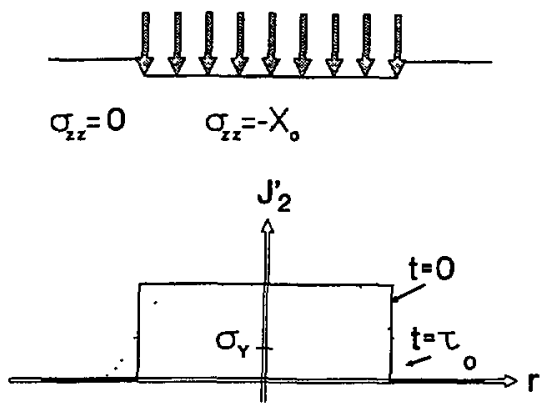

Fig. 8. - Atténuation au cours du temps de la discontinuité de $J_{2}^{\prime}$ en frontière d'impact.

[Attenuation vs. time of $J_{2}^{\prime}$ discontinuity resulting of the loading discontinuity at the impact edge.]

- A l'extérieur de l'impact, à plus d'1 mm de la frontière, le matériau n'est pas déformé plastiquement.

\section{Conclusion}

Par l'examen de la déformation à une échelle dite "cristallographique", on a pu mettre en évidence divers phénomènes d'importance lors d'un choc laser de superalliage :

- L'onde qui se propage dans le matériau présente bien un caractère d'onde de déformation uniaxiale.

- Le fort cisaillement exercé en frontière d'impact vient accroìtre l'amplitude de la contrainte équivalente $J_{2}^{\prime}$ par rapport à celle créée par l'onde plane.

- De la frontière de l'impact se développe une onde qui se propage vers l'extérieur. Un simple raisonnement par inversions de chargement montre qu'une telle onde se propage a priori aussi vers l'intérieur.

- Un surcroît de la déformation plastique cumulée, correspondant à un creux de contraintes résiduelles, s'étend sur une petite zone à partir du centre de l'impact. Ce creux de contraintes résiduelles peut s'interpréter comme le résultat d'un maximum de déformation plastique engendré lors de la propagation d'ondes de détente émises en bord d'impact. Après des premières approches simplifiées $[3,10]$ (hypothèses d'ondes de détente longitudinales seulement et à vitesse et forme données) pour la modélisation des phénomènes mis en jeu, des avancées récentes ont pris en compte la nature exacte des ondes de détente et des hypothèses plus rigoureuses [4,5]. Les résultats obtenus dans cet autre volet de l'étude corroborent les observations et interprétations cristallographiques présentées dans cet article.

Pour le praticien, l'un des intérêts principaux du choc sur Inconel 718 est de fournir immédiatement au cours des séances de tirs laser des informations sur l'effet du choc à l'échelle de la déformation microscopique, de la même manière que l'observation d'échantillons polycristallins à grains fins permet de déterminer le relief macroscopique par un simple examen approprié à l'oeil nu [3]. 


\section{Remerciements}

Les auteurs tiennent à remercier la Direction de la Recherche et de la Technologie (DRET) de la D.G.A. pour le financement de l'étude et l'autorisation de publier, la SNECMA pour la fourniture des matériaux et son soutien technique, le L.A.L.P./Arcueil et le L.U.L.I./Palaiseau pour les tirs laser, Monsieur R. Fabbro (L.A.L.P.) et Monsieur J. Fournier (à l'époque chez P.S.A.) pour la mise en œuvre du traitement et de fructueuses discussions.

\section{Bibliographie}

[1] Clauer A.H., Holbrook J.H. et Fairand B.P., Effects of Laser-induced Shock Wave Metals, in Shock Waves and High-Strain-Rate Phenomena in Metals, M.A. Meyers and L.E. Murr, Ed. (Plenum Press, New York, 1981) p. 675.

[2] Fournier J., Fabbro R., Ayrault D. et Strudel J.L., Strain Induced Microstructures by Laser Pulses, C.R. Acad. Sci. Parıs 304 (1987) 4051.

[3] Forget P., Strudel J.L., Jeandin M., Lu J. et Castex L., Laser Shock Surface Treatment of Ni-Based Superalloys, Mat. \& Manuf., Processes 5 (1990) 501.

[4] Forget P., Jeandin M. et Lyoret A., Determination of Laser Shock Treatment Conditions for Fatigue Testing of Ni-Based Superalloys, Proc. "EUROMAT '93", J. Phys. France Suppl. II3 (1993) C7-921.

[5] Forget P. et al., Modeling of Shock Wave Propagation in Laser Shock Processing (In Preparation, 1995).

[6] Décamps B., Condat M., Puig T. et Bourda C., Shearing of $\gamma^{\prime}$ Precipitates in Waspaloy Deformed by Laser Shcok, Phys. Status Soludi (a) 125 (1991) K7.

[7] Puig T., Bourda C., Thomas T., Serra J.J., Hautefeuille-Beylat L., Décamps B. et Condat M., Deformation of a $\gamma-\gamma^{\prime}$ Waspaloy After Laser Shock, Mater. Scı. Eng A 154 (1992) 183.

[8] Garcia C.I., Wang G.D., Camus D.E., Loria E.A. et Deardo A.J., Hot Deformation Behavior of Superalloy 718, in Superalloys 718, 625, 706 and Various Derivatives, E.A. Loria Ed., The Minerals, Metals \& Materials Society, 1994) p. 293.

[9] Haessner F. et Seitz W., Laser-Induced Dislocation Structures in Copper Single Crystals, J. Mat. Sci. 6 (1971) 16.

[10] Ballard P., Contraintes Résiduelles Induites par Impact Rapide. Application au Choc Laser, Thèse de Doctorat, Ecole Polytechnique (Palaiseau, France, 1991). 